Background: Systemic Lupus Erythematosus (SLE) is a prototypic autoimmune disease that characterized by the loss of self-tolerance and the production of autoantibodies (autoAbs) [1, 2]. Lupus nephritis (LN), the severe organ-threatening manifestations of SLE, could cause massive damage to patients[3, 4]. Currently, some exosomal microRNAs (miRNAs) are considered as potential biomarkers in SLE. However, the role of exosomal miRNAs in Lupus Nephritis (LN) remains unclear. Objectives: The purpose of this study was to investigate molecular mechanism of plasma exosomal miRNAs in the development of Lupus Nephritis.

Methods: Circulating exosomes were isolated from plasma of patients with LN, SLE without LN (NLN). Plasma exosomes were authenticated by Western Blot, Nanosight Tracking Analysis (NTA) and transmission electron microscopy (TEM). Fluorescence microscopy of co-cultured plasma exosomes and podocytes demonstrated that exosomes were uptaken into podocytes. Moreover, cell apoptosis and the inflammation factors was assessed using Western Blot. We analyzed the expression profiles of miRNAs in LN and NLN exosomes and the expression profiles of mRNAs of podocytes stimulated with LN and NLN exosomes with the help of next generation sequencing (NGS). Results: We demonstrate that exosomes derived from LN plasma could be taken by neighboring podocytes and promote the apoptosis of podocytes and the expression of inflammation factors. In addition, the sequencing found that miRNAs were differentially expressed in LN and NLN exosomes and mRNAs were differentially expressed in podocytes stimulated with LN and NLN exosomes.

Conclusion: LN plasma exosomes have a potency to stimulate the apoptosis of podocytes and the expression of inflammation factors. Moreover, differentially expressed miRNAs in exosomes play a potential role in the development of LN. REFERENCES:

[1] T. Colasanti, A. Maselli, F. Conti, M. Sanchez, C. Alessandri, C. Barbati, D. Vacirca, A. Tinari, F. Chiarotti, A. Giovannetti, F. Franconi, G. Valesini, W. Malorni, M. Pierdominici, E. Ortona, Autoantibodies to estrogen receptor $\alpha$ interfere with T lymphocyte homeostasis and are associated with disease activity in systemic lupus erythematosus, Arthritis and rheumatism, 64 (2012) 778-787.

[2]H.A. Al-Shobaili, A.A. Al Robaee, A.A. Alzolibani, Z. Rasheed, Antibodies against 4-hydroxy-2-nonenal modified epitopes recognized chromatin and its oxidized forms: role of chromatin, oxidized forms of chromatin and 4-hydroxy-2-nonenal modified epitopes in the etiopathogenesis of SLE, Disease markers, 33 (2012) 19-34.

[3]A. Kaul, C. Gordon, M.K. Crow, Z. Touma, M.B. Urowitz, R. van Vollenhoven, G. Ruiz-Irastorza, G. Hughes, Systemic lupus erythematosus, Nat Rev Dis Primers, 2 (2016) 16039.

[4]M.G. Tektonidou, A. Dasgupta, M.M. Ward, Risk of End-Stage Renal Disease in Patients With Lupus Nephritis, 1971-2015: A Systematic Review and Bayesian Meta-Analysis, Arthritis \& rheumatology (Hoboken, N.J.), 68 (2016) 1432-1441.

Disclosure of Interests: None declared

DOI: 10.1136/annrheumdis-2021-eular.3904

\section{POS0789 $\quad$ APPLICATION OF THE NEW ACR/EULAR 2019 CLASSIFICATION CRITERIA OF SYSTEMIC LUPUS ERYTHEMATOSUS TO A INCEPTION, MONOCENTRIC COHORT OF UNDIFFERENTIATED CONNECTIVE TISSUE DISEASES POPULATION AT ONSET OF THE DISEASE}

E. Elefante ${ }^{1,2}$, A. Parma ${ }^{1}$, V. Nannipieri ${ }^{1}$, V. Signorini ${ }^{3}$, C. Stagnaro ${ }^{3}$, D. Zucchi $^{3}$, L. Carli ${ }^{3}$, F. Ferro ${ }^{3}$, C. Tani ${ }^{3}$, M. Mosca ${ }^{3}$. ${ }^{1}$ University of Pisa, Rheumatology Unit, Department of Clinical and Experimental Medicine, Pisa, Italy; ${ }^{2}$ University of Siena, Department of Medical Biotechnology, Siena, Italy; ${ }^{1}$ University of Pisa, Rheumatology Unit, Department of Clinical and Experimental Medicine, Pisa, Italy

Background: undifferentiated connective tissue diseases (UCTDs) are a group of systemic autoimmune diseases that share clinical and serological manifestations with definite connective tissue diseases (CTDs), but do not satisfy existing classification criteria ${ }^{1}$. Within this group, it is possible to identify very heterogeneous conditions: transitory and self-limiting forms, stable conditions over time and forms that will evolve towards definite CTDs, in particular Systemic Lupus Erythematosus (SLE). The availability of new classification criteria for CTDs could be useful in identifying major CTDs from their onset and help in the differentiation from stable UCTDs, which will maintain their undifferentiated profile over time.

Objectives: the aim of this study was to apply the new ACR/EULAR 2019 classification criteria of $S L E^{2}$ to patients included, at the onset of UCTD, to evaluate how many patients could be re-classified as SLE.

Methods: this is a retrospective observational study that enrolls patients who received the diagnosis of UCTD at the Rheumatology Unit of Pisa, according to the classification criteria proposed by Mosca et $\mathrm{al}^{1}$, and were then regularly followed in the same clinic (inception cohort). For each patient, demographics, comorbidities, treatment, clinical and serological data were collected, at baseline and during follow-up. The new ACR/EULAR 2019 classification criteria of SLE were applied to the inception cohort at the onset of the disease. The characteristics of patients re-classified as SLE according to the new classification criteria and those of patients who "remained" classified as UCTD were compared.

Results: we enrolled 202 patients with a diagnosis of UCTD, mainly female (F:M 193:9) and of Caucasian ethnicity (97.5\%), mean age at the diagnosis $38,5 \pm$ 13,2 years and a median follow-up of 5 years (IQR 2-10)
During the follow-up, 10 patients $(4.9 \%)$ in our cohort developed clinical and serological characteristics that led to a diagnosis of a definite CTD, in particular: 7 SLE, 2 Mixed Connective Tissue Disease (MCTD) and 1 Sjogren's syndrome. Applying the ACR/EULAR 2019 classification criteria of SLE to patients enrolled at the onset of the disease, 38/202 (18.8\%) would have been classified as SLE (with a median score of 12 (IQR 11-15)) on the basis of the presenting clinical and serological manifestations. Interestingly, $6 / 7$ patients who received a diagnosis of SLE during the follow-up were among these 38 patients.

Among the items of the new classification criteria of SLE, the most frequently satisfied by patients "re-classified" were arthritis (63.2\%), hematological (44.7\%) and skin $(23.7 \%)$ manifestations; as for the "immunological" items, hypocomplementemia (71\%), SLE-specific (52.6\%) and antiphospholipid (35.1\%) autoantibodies Comparing the subgroup of patients "re-classified" as SLE with that of patients who "remained" UCTD, we found that the first group presented more frequently hematolog ical manifestations, hypocomplementemia and anti-dsDNA, anti-Sm, anti-RNP, antibeta2GPI positivity at the onset of the disease $(p<0.01)$. Moreover, during the follow-up the subgroup of patients "re-classified" as SLE developed more frequently malar $(p<0.05)$ and discoid rash and arthritis $(p<0.01)$ compared to patients who "remained" UCTD.

Conclusion: The development of increasingly sensible and specific classification criteria for definite CTDs may guide in the identification of stable UCTDs since their early stages and consequently in better definition of these conditions that can be considered as a distinct clinical entity.

The early identification of stable UCTD is of great importance not only for clinical management (follow-up schedules as well as therapeutic protocols) but also for scientific implications.

\section{REFERENCES:}

[1] Mosca M. et al., Clin Exp Rheumatol. Sep-Oct 1999;17(5):615-20; 2. Fanouriakis A. et al., Ann Rheum Dis. 2020 Jun;79(6):713-723.

Disclosure of Interests: None declared

DOI: 10.1136/annrheumdis-2021-eular.3909

\section{POS0790 1 RELATIONSHIPS BETWEEN SALIVA IMMUNOGLOBULINS FREE LIGHT CHAINS CONCENTRATION AND CLINICAL, IMMUNOLOGICAL, HISTOLOGICAL PARAMETERS IN PATIENT WITH PRIMARY SJOGREN'S SYNDROME}

E. Kuvardin' ${ }^{1}$, I. Kholopova², E. Belyakova ${ }^{3}$, I. Grigor'eva' ${ }^{1}$, I. Bekhtereva ${ }^{3}$, A. Maslyanskiy ${ }^{1}$, Y. Krivolapov ${ }^{3}$, S. Lapin ${ }^{2} .{ }^{1}$ Almazov National Medical Research Centre, Rheumatology Department, Saint - Petersburg, Russian Federation ${ }^{2}$ Pavlov First Saint - Petersburg State Medical University, Center for Molecular Medicine, Laboratory of Autoimmune Diagnostics, Saint - Petersburg, Russian Federation; ${ }^{3}$ North-Western State Medical University named after I.I. Mechnikov, Pathological Department of Clinical Molecular Morphology, Clinic named after E.E. Eichwald, Saint - Petersburg, Russian Federation

Background: Patients with primary Sjogren's syndrome (pSS) exhibit biological signs of $B$ cell activation such as serum polyclonal hypergammaglobulinaemia, increased levels of free light chains (FLC) and positivity for autoantibodies. B-cells may comprise up to $50 \%$ cells of lymphocytic infiltrates in salivary glands of patents with the disease. For that reasons measuring saliva B-cells products might have diagnostic value in pSS. Objectives: To evaluate relationships between saliva free light chains concentration and clinical, immunological, histological parameters in patient with pSS. Methods: Saliva and blood FLC concentrations were measured in patient with pSS $(n=24)$ and healthy volunteers $(n=11)$. Patients fulfilled the ACR/EULAR 2016 criteria of pSS. Saliva collected during assessment unstimulated salivary flow was used for analysis. Quantitative assessment of $\mathrm{CD}^{+}, \mathrm{CD}^{+}, \mathrm{CD}^{+}, \mathrm{CD}^{2} \mathrm{O}^{+}$ $\mathrm{CD}^{2} 1^{+}, \mathrm{CD}^{\circ} 8^{+}, \mathrm{CD}_{138^{+}}$cells was performed in minor salivary glands of 9 patient by immunohistochemistry. Cells quantity was calculated in $4 \mathrm{~mm}^{2}$ (area of section). Results: FLC concentration in saliva of pSS patients was higher than in healthy volunteers $(p<0,01)$. Medians of saliva $\kappa$ - and $\lambda$ - free light chains concentrations in patients and healthy persons were $1,08 \mathrm{mg} / \mathrm{L}$ [25-;75- percentiles 0,$58 ; 1,91]$, $1,038 \mathrm{mg} / \mathrm{L}[0,55 ; 2,03]$ and $0,36 \mathrm{mg} / \mathrm{L}[0,32 ; 0,54], 0,35 \mathrm{mg} / \mathrm{L}[0,21 ; 0,52] \mathrm{respec}$ tively. Median of saliva $\kappa: \lambda$ chains ratio in pSS patient was $0,94[0,6 ; 1,5]$. The ratio didn't differ from healthy persons $(p=0,24)$. Saliva $\kappa-$ and $\lambda$ - free light chain concentrations in patient with pSS correlated with patients age $\left(r_{s}=0,51, \mathrm{p}=0,033\right.$ and $r_{s}=0,43, \mathrm{p}=0,011$, respectively) and unstimulated salivary flow $\left(r_{s}=-0,48, \mathrm{p}=0,02\right.$ and $r_{s}=-0,49, \mathrm{p}=0,017$, respectively). Concentration of $\kappa-$ free light chain correlated with number of $\mathrm{CD} 138^{+}$-cells $(r=0,733, \mathrm{p}=0,025)$. Interesting that saliva $\lambda$ - free light chain concentration didn't correlate with quantity of plasmatic cells.

Conclusion: Measuring saliva FLC concentration might be offered as diagnostic marker of pSS. Concentration $\kappa-$ free light chain can directly mirror the glands quantity of plasmatic cells.

\section{REFERENCES:}

[1] Nocturne, Gaëtane; Mariette, Xavier (2018): B cells in the pathogenesis of primary Sjögren syndrome. In Nature reviews. Rheumatology 14 (3), pp. 133-145. DOI: 10.1038/nrrheum.2018.1.

Disclosure of Interests: None declared

DOI: 10.1136/annrheumdis-2021-eular.3926 